\title{
S1_File
}

\section{Risk-adjustment methods}

In general terms, the indirect risk standardization involves two steps:

Step One: Establish the risk-adjustment model: Yij f (patient characteristics), where Yij is the interested outcome for patient i from hospital j. Derive the predicted value of $\mathrm{Y}$ for each individual $(\widehat{\boldsymbol{Y} \boldsymbol{y}})$ ).

Step Two: Calculate the risk-standardized outcome measures for each hospital by:

$\left(\sum_{i=1}^{n}\right.$ Observed outcomes $/ \sum_{i=1}^{n}$ Expected outcome $){ }^{*}$ population average outcome rate.

Standard Logistic Regression:

$\mathrm{Y}_{\mathrm{ij}} \sim \operatorname{Bern}\left(1, \pi_{\mathrm{ij}}\right)$

$\operatorname{logit}\left(\pi_{\mathrm{ij}}\right)=\alpha+\beta \mathrm{X}_{\mathrm{ij}}+\varepsilon_{\mathrm{ij}}$

Hierarchical Logistic Model:

$\mathrm{Y}_{\mathrm{ij}} \sim \operatorname{Bern}\left(1, \pi_{\mathrm{ij}}\right)$

$\operatorname{logit}\left(\pi_{\mathrm{ij}}\right)=\alpha_{0 \mathrm{j}}+\beta \mathrm{X}_{\mathrm{ij}}+\varepsilon_{\mathrm{ij}}$

$\alpha_{0 j}=\alpha+\mu_{j}$

Where $i=1, \ldots, i$ is the patient level indicator, $j=1, \ldots . j$ is the hospital level indicator, $Y$ is the outcome of the patient (death/complication $=1$, survival $=0$ ), $\pi_{\mathrm{ij}}$ is the probability of death for patient $\mathrm{i}$ in hospital $\mathrm{j}$, conditional on patient-level risk factors $\mathrm{x}_{\mathrm{ij}}$. The random effect model expresses that the logit is the sum of hospital-specific intercept $\alpha \mathrm{j}$ and effects of patient-specific effects $\beta \mathrm{X}_{\mathrm{ij}}$, while the hospital intercept is a random variable with mean $\alpha$ and random variation $\mu_{\mathrm{j}} \sim \mathrm{N}\left(0, \tau^{2}\right)$.

In the standard logistic regression models, the SMR is calculated as:

$\sum_{j=1}^{n i} Y i j / \sum_{j=1}^{n i} E(Y i j \mid \alpha, \beta, X i j)$;

in the hierarchical logistic regression models, the SMR is calculated as:

$\sum_{j=1}^{n i} E\left(Y i j \mid \alpha j ; X i j, \alpha, \beta, \tau^{2}\right) / \sum_{j=1}^{n i} E\left(Y i j \mid \alpha, \beta, \tau^{2}\right)$ 